\title{
OPEN Predicting MHC I restricted T cell epitopes in mice with NAP-CNB, a novel online tool
}

\author{
Carlos Wert-Carvajal ${ }^{1,2,3,8}$, Rubén Sánchez-García, ${ }^{1,8}$, José R Macías ${ }^{1}$, \\ Rebeca Sanz-Pamplona ${ }^{4,5}$, Almudena Méndez Pérez ${ }^{1}$, Ramon Alemany ${ }^{6}$, Esteban Veiga ${ }^{1}$, \\ Carlos Óscar S. Sorzano ${ }^{1}$ \& Arrate Muñoz-Barrutia ${ }^{2,7 凶}$
}

Lack of a dedicated integrated pipeline for neoantigen discovery in mice hinders cancer immunotherapy research. Novel sequential approaches through recurrent neural networks can improve the accuracy of T-cell epitope binding affinity predictions in mice, and a simplified variant selection process can reduce operational requirements. We have developed a web server tool (NAPCNB) for a full and automatic pipeline based on recurrent neural networks, to predict putative neoantigens from tumoral RNA sequencing reads. The developed software can estimate $\mathrm{H}-2$ peptide ligands, with an AUC comparable or superior to state-of-the-art methods, directly from tumor samples. As a proof-of-concept, we used the B16 melanoma model to test the system's predictive capabilities, and we report its putative neoantigens. NAP-CNB web server is freely available at http:// biocomp.cnb.csic.es/NeoantigensApp/ with scripts and datasets accessible through the download section.

Cancer cells can accumulate many mutations that change protein sequences. It can lead to MHC-restricted T-cell epitopes ${ }^{1}$. Identifying the tumor-specific epitopes that elicit $\mathrm{T}$ cell cytotoxic responses represents a major challenge for cancer immunotherapy, particularly to design personalized therapies ${ }^{1,2}$. Finding neoantigens in every cancer patient will be fundamental for the next generation of antitumor immunotherapies.

A plethora of neoantigen discovery pipelines has been described to enable the prediction of epitopes from genetic information. However, current pipelines are human-centered and, thus, are primarily designed for clinical usage ${ }^{3,4}$. Among the preeminent research lines, genomic analysis adjustments ${ }^{3,5-8}$, and neoepitope ranking practices ${ }^{5,6,8,9}$ have been prioritized over affinity binding or immunogenicity prediction algorithms. Despite this, the latter ones remain a critical component of the overall workflow for which limited available options exist ${ }^{10}$.

The absence of dedicated tools for the alternative in vivo mouse models hinders pre-clinical cancer immunotherapy research. Hence, laboratories have to produce or adapt to ad-hoc human pipelines. The pipelines Epi-Seq ${ }^{11}$, pVAC-Seq $^{3}, \mathrm{MuPeXI}^{9,12}$ and Neoantimon ${ }^{13}$ offer modified versions for the murine model. These platforms follow the canonical prediction process, based on sequencing data to estimate the gene expression and the predicted affinity with the T-cell receptor (TCR) of the mutated peptide ${ }^{10}$, which is a prerequisite to elicit an immune response ${ }^{1}$. Epi-Seq performs a full-analysis from DNA and RNA reads file, however, it is not tailored for neoantigen detection, as it was conceived for the discovery of common tumor antigens. The other platforms lack genome preprocessing and variant calling in its analysis. Hence, in these three options, a variant call format file (VCF) its needed for its usage. Among them, solely MuPeXI is accesible as a webserver whilst pVAC-Seq and Neoantimon have to be installed locally and require a BAM file to estimate the levels of gene expression, which underscores the importance of a comprehensive and integral pipeline as a freely accessible webservice.

\footnotetext{
${ }^{1}$ Centro Nacional de Biotecnología, Consejo Superior de Investigaciones Científicas, 28049 Madrid, Spain. ${ }^{2}$ Departamento de Bioingenieria e Ingenieria Aeroespacial, Universidad Carlos III de Madrid, 28911 Leganés, Spain. ${ }^{3}$ Bioengineering Department, Imperial College London, London SW7 2AZ, UK. ${ }^{4}$ Unit of Biomarkers and Susceptibility, Oncology Data Analytics Program (ODAP), Catalan Institute of Oncology (ICO), Oncobell Program, Bellvitge Biomedical Research Institute (IDIBELL), 08908 L'Hospitalet de Llobregat, Spain. ${ }^{5}$ Centro De Investigación Biomédica en Red de Epidemiología y Salud Pública (CIBERESP), Madrid, Spain. ${ }^{6}$ Procure Program, Institut Català d'Oncologia- Oncobell Program, Catalan Institute of Oncology (ICO), Oncobell Program, Bellvitge Biomedical Research Institute (IDIBELL), 08908 L'Hospitalet de Llobregat, Spain. 'Instituto de Investigación Sanitaria Gregorio Marañón (IiSGM), 28007 Madrid, Spain. ${ }^{8}$ These authors contributed equally: Carlos Wert-Carvajal and Rubén Sánchez-García. ${ }^{\boxplus}$ email: mamunozb@ing.uc3m.es
} 
The algorithms underpinning the prediction of immune response differ aming these options. Epi-Seq and MuPeXI use NetMHCPan ${ }^{14}$ and its pan-specific variant, NetH2 pan $^{15}$, which rely on dense neural networks for binding affinity prediction. These tools have been trained with samples from the major histocompatibility complex (MHC) of mice or H-2. pVAC-Seq and Neoantimon also include MHCflurry ${ }^{16}$, which recently has been upgrated to include an estimation of immunogenicity through an antigen processing model using a convolutional neural network. In general, among the supervised machine learning methods that have facilitated the identification of neoepitopes, artificial neural networks have proven to be highly efficient ${ }^{17}$. However, recurrent neural networks (RNN) remain quite unexplored even if they are better suited for sequential problems, as attested by their extensive usage in natural language processing systems ${ }^{18}$. As a case, long short-term memory (LSTM) units are, at present, used for protein prediction of function and interactions ${ }^{19,20}$.

Prediction models have relied on gene expression information from tumor samples to determine putative peptides for intervention ${ }^{1}$. However, current approaches depend on genetic information from DNA sequencing to determine mutations ${ }^{5,8}$. This dependence hinders temporal performance and increases intervention costs, but whole-exome sequencing (WES) is justified for its improved selectivity ${ }^{21}$. Hence, a system may rely exclusively on RNA sequencing (RNA-Seq) to simultaneously identify mutations and gene expression levels ${ }^{21}$. If compensatory methods in neoepitope prediction are present, a tool designed for pre-clinical use may only rely on mutational information from RNA-Seq for a cost-effective solution. We developed an integrated pipeline optimized for a murine model that finds putative neoepitope via next-generation sequencing (NGS) tumor variant calling and ranks them using LSTMs. This novel platform is only based on RNA-Seq, and is automated for a given haplotype. As a proof-of-concept, we trained our system with the $\mathrm{H}-2 \mathrm{~K}^{\mathrm{b}}$ haplotype (MHC class I) to be tested for the commonly used B16 melanoma model in C57BL/6 mice, but the tool is compatible with additional typings that correspond to the most common in $\mathrm{C} 57 \mathrm{BL} / 6^{22}$ and $\left.\mathrm{FVB} / \mathrm{N}\right)^{23,24}$.

Furthermore, the NAP-CNB is available separately as sequence affinity binding predictor. Entries are also constrained by a minimum length for each haplotype as tool is conceived for a NGS-based analysis in which proteins are submitted in their full extension. The resource NAP-CNB is freely available as a web server at http:// biocomp.cnb.csic.es/NeoantigensApp/.

\section{Methods}

The proposed pipeline employs genome preprocessing tools, variant calling software, and customized neural network architecture to obtain putative neoantigens from RNA-Seq experiments. As an integrative tool, the workflow has been adapted into a web server for RNA-Seq file submissions with filtering options available at the preprocessing level, as shown in Fig. 1a. A tumor RNA-Seq file should be inputted as ".fastq.gz" together with the MHC class I type and an email address to receive the final results in less than ten hours. The binding affinity predictor is also available separately to be used for peptides sequences in FASTA format, which is able to process 5000 sequences in less than 30 seconds.

Variant calling: from RNA-Seq to mutant peptides. The somatic mutations suitable for neoantigen prediction are obtained from the gene expression of tumor tissue (RNA-Seq). NGS technologies that produce a FASTQ file are required for this protocol.

First, a quality assessment report is produced using FastQC (v0.11.8) ${ }^{25}$ for user evaluation. In terms of preprocessing, the RNA-Seq file is realigned with a reference genome for further processing with STAR (v2.6.0a) ${ }^{26}$. The resulting BAM file is processed with Picard (v2.19.2) ${ }^{27}$ for further refinements such as annotation and duplicate marking. Subsequently, Genome Analysis Toolkit (GATK, v4.1.2.0) ${ }^{28}$ is used for exon segmentation, through the "SplitNCigarsReads" protocol, and base quality score recalibration (BQSR) following Best Practices guidelines $^{29}$. As indicated in Fig. 1b, this part serves as a preprocessing of the RNA-Seq reads per se before variant calling. At this level, the user may introduce more flexible or conservative restrictions at the quality level by modifying the default threshold of BQSR.

The MuTect2 variant caller ${ }^{30}$ from the GATK package is used in its tumor-only mode (Fig. 1b), which is computationally less expensive but provides a higher number of false positives ${ }^{31}$. Even if designed primarily for DNA-Seq reads, MuTect2 has shown to be efficient in calling mutations from RNA-Seq ${ }^{32}$. By default, tumoral RNA-Seq is matched with databases of single nucleotide polymorphisms (dbSNP), although it can be used with a panel-of-normals (PoN) by construction. Following depth coverage (DP) filtering, the variants are submitted to Variant Effector Predictor (VEP) from Ensembl (v100.0) ${ }^{33}$ for annotation and extraction of mutant peptide sequences identified as missense variants. An additional allele frequency (AF) can be introduced at submission. Finally, a script matches the resulting UniParc reference from VEP to extracted UniProt proteins for proteinlevel prediction ${ }^{34}$.

Additionally, Cufflinks (v2.2.1) ${ }^{35}$ is used for mRNA abundance estimation as measured by fragments per kilobase million (FPKM). As there is no range for optimal neoantigen expression, this metric is provided to the user for its examination (Fig. 1b).

Hence, NAP-CNB provides a simplified interface for users to submit neoepitope prediction jobs to a webserver. Hence, it removes the need for a local machine, as required by Epi-Seq ${ }^{11}$, pVAC-Seq $^{3}$ and Neoantimon ${ }^{13}$ and, in contrast with MuPeXI ${ }^{9,12}$, it additionally provides variant calling capabilities. Nonetheless, current customization remains limited. The output consists of a list of sequences with a softmax score and a complementary binary metric from postprocessing. Additionally, levels of expression are also included for the user. Jobs can be downloaded as lists or ".csv" files, which permits easy analysis and compatibility with data analysis software to perform further candidate sorting and selection. 


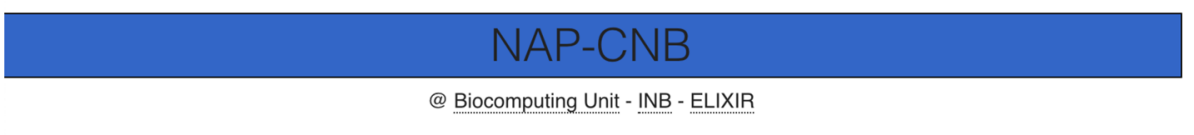

\begin{tabular}{l|l|}
\hline Email: \\
\hline \\
Haplotype: \\
\hline H-2Kb
\end{tabular}

Base quality score threshold (BQSR>):

18

Maximum depth coverage (DP>):

30

Minimum allele frequency (AF<):

(Af

Cancer file:

Seleccionar archivo Ningún archivo seleccionado

NAP-CNB was developed and is maintained at the Biocomputing Unit - CNB by Carlos Wert-Carvajal carloswertcarvajal@gmail.com. Citing the web-server?

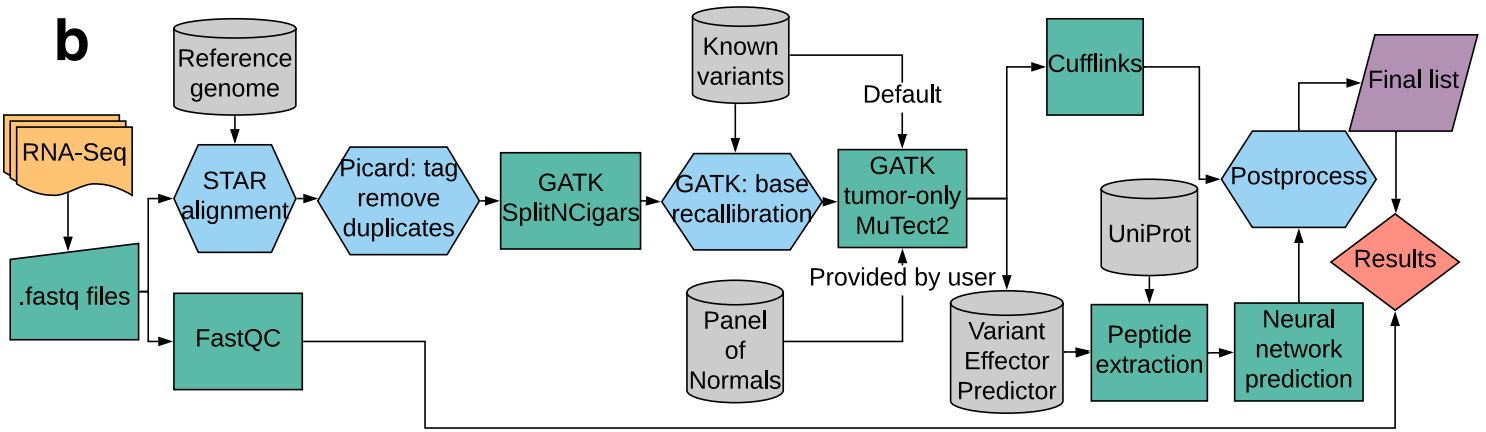

Figure 1. Workflow for the integrated pipeline. (a) The user interface of NAP-CNB with the fields required for NGS analysis. Users can introduce filters of GATK for base quality score recallibration (BQSR) of RNASeq reads, minimum depth coverage (DP) and allele frequency (AF). Additionally, users may submit peptidic sequences for affinity prediction. Individual submissions are haplotype-specific, and results are sent to an email address. (b) Workflow for the integrated pipeline. Firstly, the sample is preprocessed before variant calling. Quality control through FastQC and STAR alignment with the reference genome is followed with protocols from Best Practices of GATK. Known variants are introduced through known polymorphisms or a panel-ofnormals if requested, andsufficient non-tumor RNA-Seq reads are provided. MuTect 2 is used for variant calling, and plausible single nucleotide variant (SNV) mutations translated into peptidic sequences for prediction with the RNN model. Gene expression is quantified through Cuffquant in Cufflinks.

Dataset generation and preprocessing. Sequences of MHC-I binding peptides were obtained from the IEDB database ${ }^{36}$ for the $\mathrm{H}-2 \mathrm{D}^{\mathrm{b}}, \mathrm{H}-2 \mathrm{D}^{\mathrm{d}}, \mathrm{H}-2 \mathrm{D}^{\mathrm{q}}, \mathrm{H}-2 \mathrm{~K}^{\mathrm{b}}, \mathrm{H}-2 \mathrm{~K}^{\mathrm{d}}, \mathrm{H}-2 \mathrm{~K}^{\mathrm{q}}, \mathrm{H}-2 \mathrm{~L}^{\mathrm{d}}$ and $\mathrm{H}-2 \mathrm{~L}^{\mathrm{q}}$ haplotypes, although here we present the procedure and results of $\mathrm{H}-2 \mathrm{~K}^{\mathrm{b}}$ as a case. Given the different binding assessment methodologies considered in IEDB, elements were binarized by their MHC class I classification as positive or negative, per IEDB standards. The datasets, by entries accession number, are available at NAP-CNB.

Firstly, peptides deemed as antigenic were processed to extract their binding sites. These correspond to positive epitopes from IEDB as classified by their qualitative labels "Positive High", "Positive Intermediate" and "Positive Low" for each MHC class I haplotype in mice irrespective of the assay type. A further selection criteria was to include only epitopes with protein identifications to generate negatives and resize the sequence to a given length. Consequently, sequences were aligned with its protein source through the Smith-Waterman algorithm ${ }^{37}$ to obtain the remaining sequence as negative samples (Suppl. Fig. 1). Additionally, epitope regions were extended through the original sequence to have a regular size (Suppl. Fig. 1). In contrast with previous methods, a given prevalence (i.e., the fraction of the minority class) was not imposed on the dataset. In total, for $\mathrm{H}-2 \mathrm{~K}^{\mathrm{b}}, 4,828$ 
peptide entries were processed into 251,049 sequences with 6714 positive entries and 244,225 negatives. A $10 \%$ split was used for test set generation. Concerning blind test data, IEDB datasets 1034799 and 1035276 were processed through the previous procedure and by the method described by ${ }^{15}$. Additional information concerning the dataset for each haplotype is available in the download section of NAP-CNB.

Further postprocessing was implemented with a majority vote algorithm that considered mutations to the most similar amino acid, given by the BLOSUM62 matrix $^{38}$, for each position. In other terms, a sequence modified its classification if there was a consensus among its most akin peptides.

Neural network training. The neural networks were implemented through Keras (v2.2.4) ${ }^{39}$ and TensorFlow (v1.11.0 $)^{40}$. A scalable routine was used for architecture optimization through simplified datasets (Suppl. Fig. 1) until one competent was obtained. Moreover, training was done with "on-batch" class balancing and data augmentation. The latter increased the number of positives sequences through random substitution of a given number of amino acids with similar ones from the BLOSUM62 matrix $^{38}$, with a given tolerance (Suppl. Fig. 3). The training was performed through fivefold cross-validation, for hyperparameters tuning and optimization of balancing and augmentation, generating a total of 80 models for the actual dataset.

The initial toy model was used for embedding selection and tuning of neural architectures (Suppl. Table 1A,B), which was maintained in the type and depth of layers in later configurations. At this stage, there were no significant improvement in any of three low-dimensional embeddings ${ }^{41-43}$, against a one-hot encoding (Suppl. Table 1A). Hence, we maintained the dimensions given by the naturally occurring amino acids. While an intermediate dataset (Suppl. Fig. 1C) was introduced for data balancing and augmentation. The final model was produced with the complete dataset and cross-validation of the number of internal LSTM units at each layer, the number of on-batch sequence augmentations, and its tolerance, and the on-batch class balancing.

In the final architecture, peptide sequences of a given length are introduced with a one-hot encoding representation to three consecutive bidirectional LSTM layers, followed by three layers of dense neurons with two intermediate dropouts units. The output layer consists of a dense neuron, with a soft-max activation, which yields the affinity estimation probability. The overall network is represented in Fig. 2.

Sequencing raw data. An in vitro B16 melanoma cell line with a $\mathrm{H}-2 \mathrm{~K}^{\mathrm{b}}$ haplotype was processed for RNA extraction and sequenced through an NGS Illumina HiSeq2000. From the FastQC analysis, all evaluated parameters were satisfactory except from the presentation of four over-represented sequences corresponding to Illumina single end PCR primer and technical noise as TrueSeq adaptors. Trimming of these sequences was done before RNA-Seq processing. The resulting “.fastq.gz" file was introduced for analysis in a local server.

\section{Results}

Cross-validation metrics. Initial architectures, based on LSTM and dense layers, showed performance improvements, in terms of the area under the curve for the receiver operator characteristic (AUC ROC), for higher depth models (Suppl. Table 1A). Despite this, these changes did not have an impact as significant as "onbatch" balancing and data augmentation. In particular, modifications of a "virtual" prevalence raised AUC ROC and F-1 values to $20 \%$ in test sets (Suppl. Table 1C) and decreased the degree of overfitting. All parameters were adjusted through grid search on the final model under a limited number of epochs (see Additional file 2-Grid search parametrization). As observed in Table 1, the network's final AUC ROC for $\mathrm{H}-2 \mathrm{~K}^{\mathrm{b}}$ reached $95 \%$, albeit with an acceptable F1 score, due to the assumed low prevalence. The complete cross-validation results of each model are available at NAP-CNB. For further evaluation in the $\mathrm{H}-2 \mathrm{~K}^{\mathrm{b}}$ haplotype, $10 \%$ of the original dataset was used as a test set of the selected parametrized system. In Fig. 3, both the ROC and the precision-recall curve are shown. The latter reflects how the system fares against a high-class imbalance. In terms of metrics, the ROC AUC for the test sample was $86.5 \%$ with $97.2 \%$ accuracy. Notwithstanding, the proposed ensemble method for postprocessing could increase precision by $7.6 \%$. Throughout cross-validated models, window sizes of 8,10 , and 12 amino acids were tested for predictive performance. Sequences of 12 amino acids produced more accurate models (Fig. 4). This result may indicate that antigenic determinants are not sufficient for peptide classification and distal amino acids carry additional predictive information. The distribution of sequences classified as positive and a sensitivity analysis from random classifications showed similar results (Suppl. Fig. 4). In contrast, $\mathrm{NetH} 2$ pan has reported a greater accuracy for short sequences around epitopes ${ }^{15}$.

The cross-validation metrics of the all generated haplotypes presents both enhancements and reductions in efficacy, as shown in Table 2 . In the typings $\mathrm{H}-2 \mathrm{~K}^{\mathrm{d}}, \mathrm{H}-2 \mathrm{~K}^{\mathrm{k}}$ and $\mathrm{H}-2 \mathrm{~L}^{\mathrm{q}}$ the best performance corresponded to 8 -mers. We provide, as an example of further benchmarking and binary metrics, additional results for $\mathrm{H}-2 \mathrm{~K}^{\mathrm{d}}$ (Suppl. Material. H2-Kd). Moreover, for this typing, we report a suboptimal cross-prediction with $\mathrm{H}-2 \mathrm{~K}^{\mathrm{b}}$ (Suppl. Material. H2-Kd), which evidences the need for individual networks for each haplotype.

Benchmarking. In contrast with NetH2 pan $^{15}$, which is the benchmark used for MHC class I affinity prediction in mice, the reported cross-validated AUC ROC, in Table 2, were comparable or superior with a $95 \%$ for $\mathrm{H}-2 \mathrm{~K}^{\mathrm{b}}$, which is $3 \%$ higher, and a similar performance in PPV. Results vary for each haplotype and we report a hindered efficiency in some haplotypes such as $\mathrm{H}-2 \mathrm{D}^{\mathrm{b}}$. Results of binding affinity are also on par with those from MHCflurry $2.0^{16}$, showing improved scores for $\mathrm{H}-2 \mathrm{~K}^{\mathrm{k}}$ and a worsening for $\mathrm{H}-2 \mathrm{~L}{ }^{\mathrm{q}}$, for instance. MHCflurry 2.0 does provide a more refined metric for immunogenicity by predicting antigen processing.

The divergence in the generation of negatives and the assumed prevalences may render the comparison in cross-validation metrics with both methods insufficient. Hence, to confirm a better performance against $\mathrm{NetH} 2$ pan on a dataset, blind testing was implemented from two new $\mathrm{H}-2 \mathrm{~K}^{\mathrm{b}}$ datasets from IEDB (1034799 and 1035276). Negatives were generated following the protocol mentioned above, disregarding positive sequences 


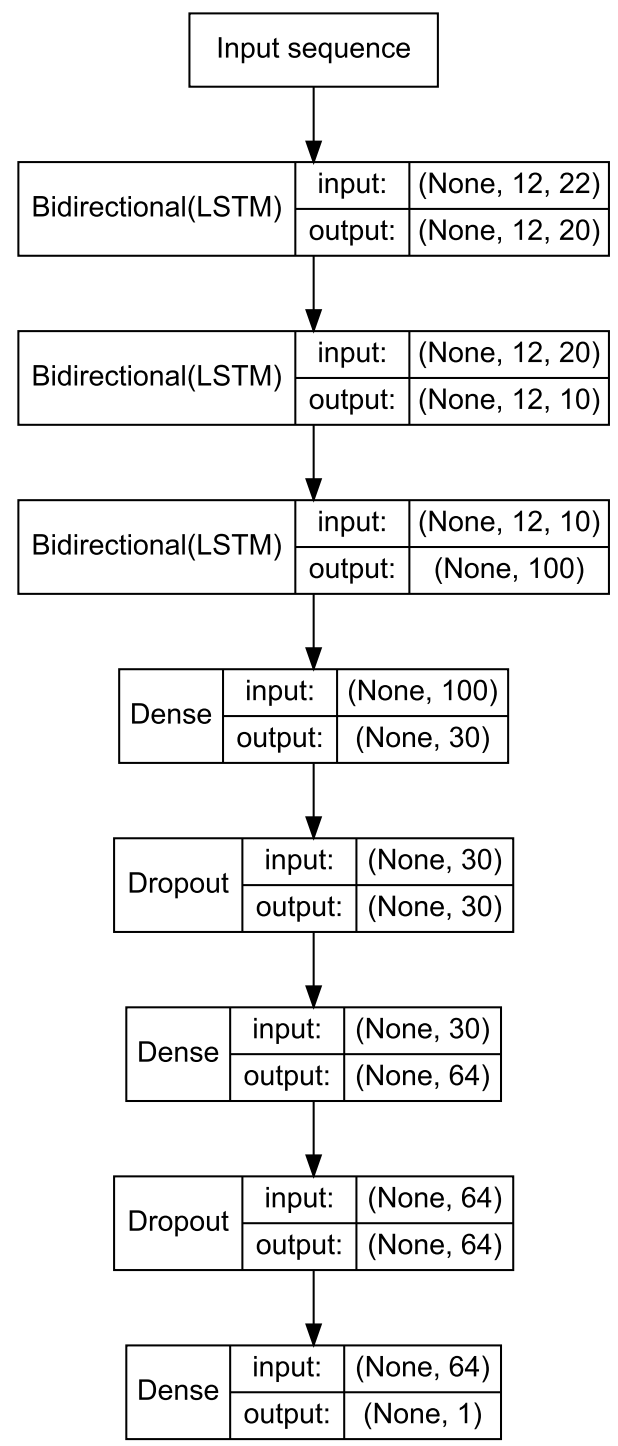

Figure 2. Neural network model of the binding affinity prediction for $\mathrm{H}-2 \mathrm{~K}^{\mathrm{b}}$. The input sequence corresponds to a one-hot encoding of a 12 mer peptide sequence extracted from the preprocessing workflow. The number of LSTM units corresponds to the input sequence's overall length across the three consecutive layers. Following the RNN, two hidden dense units, with alternating dropouts, serve to process an affinity probability.

that do not have a protein accession or cannot be reframed into 12-mers, and by generating random sequences with an assumed prevalence as described in NetH2 pan $^{15}$. Given that NetH2pan considers different epitope lengths and substitutions, binarization was done by considering whether binds were predicted overall for a 12 mer sequence. Even if this size was chosen for an evaluation under equal conditions, it should be noted that NetH2pan predicts better shorter sequences on average (Suppl. Fig. 5). In all binary metrics, the LSTM network achieved improved results (Suppl. Figs. 6 and 7). The reported accuracies for were between $96 \%$ and $98 \%$, with up to threefold increases in precision.

Notably, in all cases, positives were better detected than in NetH2pan for 12 mers irrespective of the method used to produce negative sequences. On the whole, our approach detected 259 and NetH2pan 86 of a total of 438 antigens across both datasets. Moreover, an ensemble method joining predictive positives from both methods improved detection to 277 with random negatives and 254 with negative sampling.

Use case. As a result of MuTect 2 calling, 4566 variants were identified. From those, 1085 missense transcripts were obtained from VEP corresponding to 345 genes. These were matched against the results from Cufflinks and submitted for prediction. In the end, our proposed software generated a ranking of putative neoantigens. The 35 top-scoring putative neoepitopes are shown in Table 3. The predictions were matched with the original B16 results from Castle et al. ${ }^{44}$ (Suppl. Table 2). Additionally, we compared the rank given by our proposed algo- 


\begin{tabular}{|l|l|l|l|l|l|}
\hline AUC ROC & ACC & PPV & Sensitivity & Specificity & F1 \\
\hline$( \pm$ SD $)$ & $( \pm$ SD $)$ & $( \pm$ SD $)$ & $( \pm$ SD $)$ & $( \pm$ SD $)$ & $( \pm$ SD $)$ \\
\hline $0.95 \pm 0.04$ & $0.977 \pm 0.004$ & $0.6 \pm 0.1$ & $0.62 \pm 0.09$ & $0.988 \pm 0.004$ & $0.6 \pm 0.1$ \\
\hline
\end{tabular}

Table 1. Binary classification metrics for the final fivefold cross-validated algorithm for the $\mathrm{H}-2 \mathrm{~K}^{\mathrm{b}}$ typing. The reported mean statistics estimators correspond to AUC ROC, accuracy (ACC), precision or positive predictive value (PPV), and sensitivity and specificity with their harmonic average (F1). The prevalence of positive samples was around 1:40.
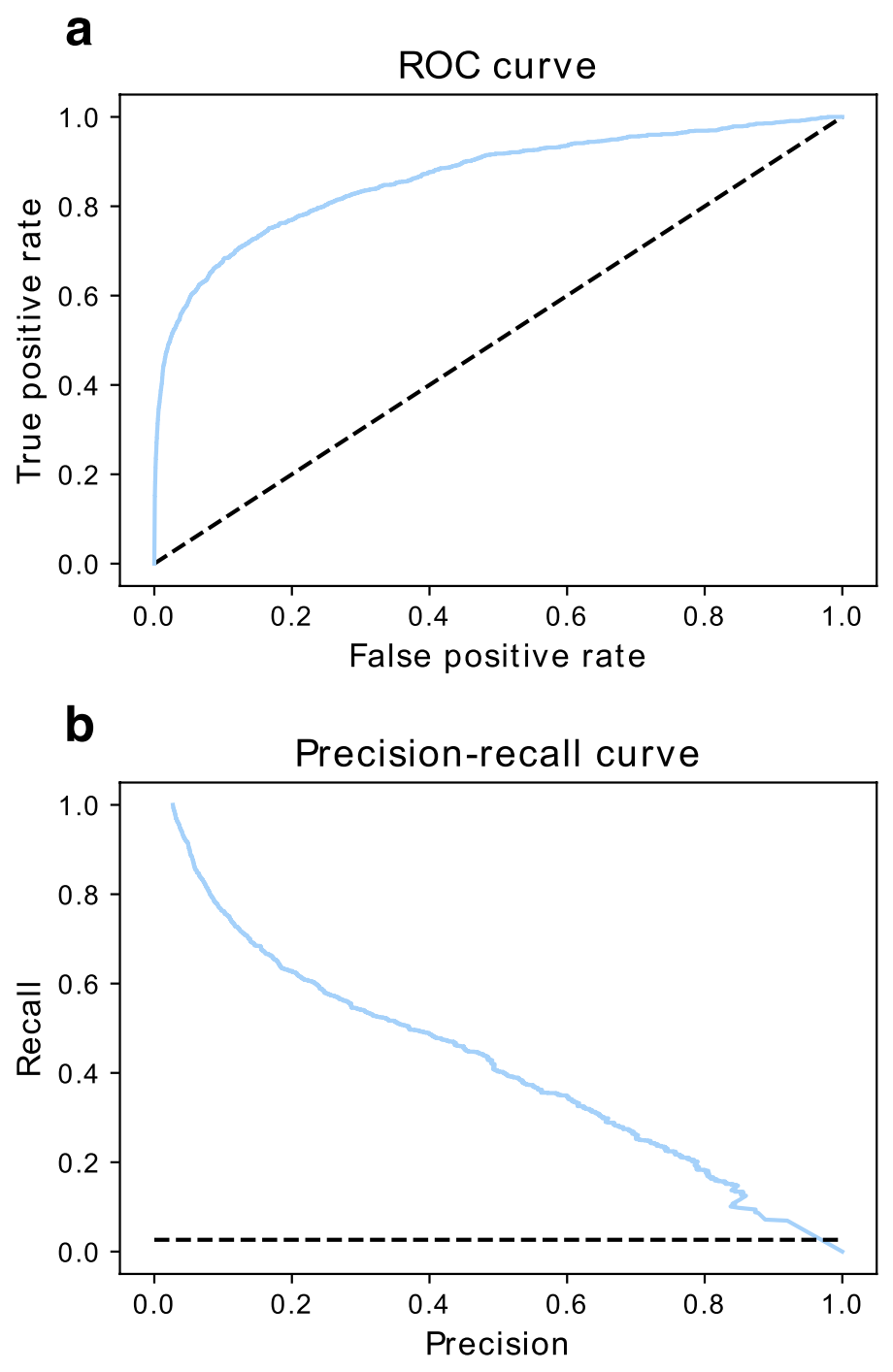

Figure 3. ROC and precision-recall curves for the final model trained with $\mathrm{H}-2 \mathrm{~K}^{\mathrm{b}}$ samples. (a) ROC curve for $10 \%$ test partition with an AUC of 86.5\%, the dashed line shows chance level. (b) Precision-recall curve with the prevalence of around $3 \%$ shown as chance. The precision-recall AUC is $41.97 \%$, whereas a random guess corresponds to an AUC of $2.64 \%$ for the same data imbalance.

rithm's softmax score with the relative classification of the 12 mer sequence in NetH2pan ${ }^{15}$ and MHCflurry $2.0^{16}$, obtained by averaging the scores across all of its possible epitope lengths and mutations. Table 3, thus, establishes an order of preference for both methods. Due to sample size limitations, the haplotype $\mathrm{H}-2 \mathrm{D}^{\mathrm{b}}$ of the C57BL/6 model is not analyzed but should also be included in a naïve study.

From an implementation perspective, NAP-CNB simplifies the overall process in comparison with previous murine pipelines by removing the need of performing variant calling separately. In terms of overall performance, the entire pipeline has an execution time of around ten hours in a local server using two CPU cores. This duration 


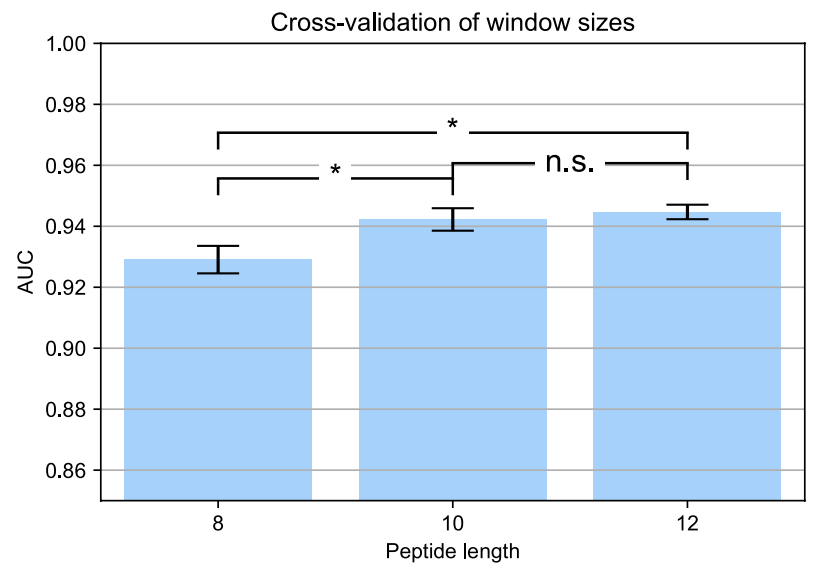

Figure 4. Cross-validation of peptide window sizes for $\mathrm{H}-2 \mathrm{~K}^{\mathrm{b}}$. The area under the curve of the receiver operating characteristic curve using 8 mers, 9 mers, and 12 mers obtained through fivefold cross-validation in different conditions. The windows are obtained from the mutated peptide sequence centered at the location of the SNV. Significant differences between means (Student's t-test, $\mathrm{p}<0.05$ ) are shown.

\begin{tabular}{|l|l|l|}
\hline Haplotype & AUC ROC $( \pm$ SD) & Peptide length (mer) \\
\hline $\mathrm{H}-2 \mathrm{D}^{\mathrm{b}}$ & $0.7 \pm 0.1$ & 12 \\
\hline $\mathrm{H}-2 \mathrm{D}^{\mathrm{d}}$ & $0.9 \pm 0.1$ & 12 \\
\hline $\mathrm{H}-2 \mathrm{D}^{\mathrm{q}}$ & $0.8 \pm 0.1$ & 12 \\
\hline $\mathrm{H}-2 \mathrm{~K}^{\mathrm{k}}$ & $0.96 \pm 0.06$ & 8 \\
\hline $\mathrm{H}-2 \mathrm{~K}^{\mathrm{q}}$ & $0.9 \pm 0.2$ & 12 \\
\hline $\mathrm{H}-2 \mathrm{~L}^{\mathrm{d}}$ & $0.9 \pm 0.1$ & 12 \\
\hline $\mathrm{H}-2 \mathrm{~L}^{\mathrm{q}}$ & $0.7 \pm 0.2$ & 8 \\
\hline
\end{tabular}

Table 2. AUC ROC scores and minimum required peptide lengths of haplotypes implemented in NAP-CNB. The AUC ROC corresponds to the fivefold cross-validation average of the best configuration obtained through grid-search parametrization. In all haplotypes 128 models were initially generated for lengths of 8,10 and 12 amino acids with additional fine-tuning for some instances.

corresponds to steps between preprocessing of the RNA-Seq and quality analysis to affinity prediction. The levels of abundance are presented to guide the user in selecting a candidate.

\section{Discussion}

The proposed pipeline provides an integrated software solution for mouse neoantigen MHC class I discovery from RNA-Seq data. The workflow is based on a streamlined process adapted to the resource-efficient and accessibility requirements of pre-clinical research. Notably, we report an affinity binding estimation model that successfully improves previously reported performance. The B16 case study also shows a good number of putative neoantigens that are coherent with literature estimates ${ }^{44}$. A functional validation measuring T-cell immune responses by ELISPOT or intracellular IFN-gamma staining in mice responding to B16 tumors would be required to validate the prediction results.

In terms of the actual prediction algorithm, the RNN-based approach presents an AUC ROC of $95 \%$ in crossvalidation. Compared with the current NetH2pan benchmark model ${ }^{15}$, it represents an enhancement in terms of accuracy and precision for the $\mathrm{H}-2 \mathrm{~K}^{\mathrm{b}}$ haplotype in both cross-validation and blind testing metrics, with a threefold increase of precision in the latter. However, this varies depending on the haplotype used, with $\mathrm{H}-2 \mathrm{~K}^{\mathrm{d}}$, for instance, lacking such improvements for a blind set. Additionally, this approach eludes a more refined version of immunogenicity prediction as the one presented by MHCflurry $2.0^{16}$, although it presents a comparable performance in their binding affinity estimation. Thus, these results may reinforce sequential models' usefulness as an efficient solution to antigen binding prediction against more conventional neural network approaches. Future lines of research may include more recent sequential model innovations. Novel types of sequential architectures in transformers and RNNs, such as BERT ${ }^{45}$ and GORU ${ }^{46}$, could serve as enhancers of overall performance. Also, subsequent work in epitope size should aim to reconcile flexibility, which is compatible with an RNN-based framework, with the generation of empirical negative samples. The web server restricts the haplotype utilized for prediction. Even if cross-prediction between haplotypes $\mathrm{K}^{\mathrm{b}}$ and $\mathrm{K}^{\mathrm{d}}$ suggests type-specific modeling is an optimal solution, a pan-specific system is part of the future directions.

Concerning data processing, the use of negative empirical sequences and data augmentation should also be considered to improve affinity estimation. Strategies could include generative models such as Gaussian mixtures 


\begin{tabular}{|c|c|c|c|c|c|c|c|}
\hline Rank & Sequence & Gene & Probability & FPKM & Castle et al. & NetH2pan & MHCflurry 2.0 \\
\hline 1 & NKVVMEYENLEK & Pnp & 1.00 & 3.04 & - & 24 & 22 \\
\hline 2 & KASGFRYNVLSC & Nr1h2 & 1.00 & 0.00 & - & 1 & 17 \\
\hline 3 & SQAWTHPPGVVN & Adar & 1.00 & 0.00 & - & 88 & 128 \\
\hline 4 & TFVYPTIFPLRE & Lrrc28 & 1.00 & 0.94 & - & 10 & 14 \\
\hline 5 & DKSYTLPSSLRK & Zic2 & 1.00 & 1.83 & - & 27 & 28 \\
\hline 6 & TLAQLTWPLWLE & Hjurp & 0.43 & 0.00 & - & 26 & 72 \\
\hline 7 & VDTNMMGHEHIR & Safb2 & 0.26 & 24.20 & - & 140 & 150 \\
\hline 8 & AKTAVNDYFQCN & Stox 2 & 0.25 & 0.00 & - & 126 & 179 \\
\hline 9 & FIAIYHHASRAI & Tm9sf3 & 0.21 & 24.29 & ** & 8 & 40 \\
\hline 10 & SGASNTTPHLGF & Tab2 & 0.20 & 29.21 & - & 103 & 58 \\
\hline 11 & YSSMRMMKEALQ & Herc6 & 0.18 & 10.93 & - & 38 & 102 \\
\hline 12 & TRASVTNFQIVH & Tulp2 & 0.16 & 0.00 & - & 43 & 16 \\
\hline 13 & AWGVDGTLAQLE & Pkdcc & 0.16 & 5.50 & - & 118 & 134 \\
\hline 14 & VVLLMDALYLLR & Sirpa & 0.14 & 51.24 & - & 13 & 49 \\
\hline 15 & NVTISNLYEGMM & Hjurp & 0.13 & 0.00 & - & 6 & 20 \\
\hline 16 & ARALWFWAFSLQ & Sfil & 0.09 & 0.00 & - & 5 & 47 \\
\hline 17 & GASSFREAMRIG & Eno3 & 0.09 & 29.01 & - & 21 & 112 \\
\hline 18 & LAAIVGKQVLLG & Rpl13a & 0.09 & 1203.49 & * & 67 & 5 \\
\hline 19 & AYSAHTSENLED & Zfp638 & 0.09 & 0.00 & - & 142 & 181 \\
\hline 20 & TVAVLGFILSSA & Commd4 & 0.09 & 41.28 & - & 52 & 30 \\
\hline 21 & FQYCLFKICRDV & \begin{tabular}{|l} 
Pla2g12a \\
\end{tabular} & 0.08 & 7.05 & - & 63 & 101 \\
\hline 22 & AISAPCIGSPGC & Hjurp & 0.08 & 0.00 & - & 227 & 297 \\
\hline 23 & HKHLMPTQIIPG & Jmjdlc & 0.08 & 3.42 & - & 144 & 106 \\
\hline 24 & MFGIDGFAAVIN & Pdhx & 0.07 & 10.26 & - & 56 & 59 \\
\hline 25 & YQPRQSVSYEDV & Tasor2 & 0.06 & 5.16 & - & 188 & 220 \\
\hline 26 & LCPLESRVPHTL & Hjurp & 0.06 & 0.00 & - & 218 & 127 \\
\hline 27 & QMIVFYLIELLK & Jak2 & 0.05 & 6.03 & - & 2 & 6 \\
\hline 28 & AHMYEAVALIKD & Dennd5a & 0.05 & 64.21 & - & 17 & 9 \\
\hline 29 & DRIVHALNTTVP & Ccdc58 & 0.05 & 0.00 & - & 70 & 108 \\
\hline 30 & NEVDVQEVTHSA & $\operatorname{Dlg} 4$ & 0.04 & 9.45 & - & 289 & 138 \\
\hline 31 & LAAIVGKQVLLV & Rpl13a & 0.04 & 1203.49 & * & 48 & 2 \\
\hline 32 & QRNRKLDYSSSE & Bod1l & 0.04 & 3.65 & - & 282 & 328 \\
\hline 33 & HLGCIKKKFLQR & Sfil & 0.04 & 0.00 & - & 177 & 225 \\
\hline 34 & PPTARMMFSGLA & Wiz & 0.03 & 16.70 & - & 18 & 167 \\
\hline 35 & QEEVFAKHVSNA & Smarcc2 & 0.03 & 0.00 & - & 167 & 104 \\
\hline
\end{tabular}

Table 3. Putative neoantigens, shown by sequence and gene symbol, ranked by scores for the $\mathrm{H}-2 \mathrm{~K}^{\mathrm{b}}$ restricted $\mathrm{B} 16$ melanoma model. The gene expression is quantified as fragments per kilobase million. Neoantigens examined in Castle et al. ${ }^{44}$ are classified by selection for validation $\left(^{*}\right)$ and reactivity $\left(^{* *}\right)$. Ranked classification of the average scores of peptide sequences for a complete 12 mer sequence, considering epitope lengths between 8 and 12, given by NetH2pan and MHCflurry 2.0. The ranking of NetH2pan and MHCflurry 2.0 corresponds to binding affinity and presentation scores, respectively.

or adversarial networks (GAN) ${ }^{47}$. Nonetheless, one of the problems posed by the dataset is its reliance on a binarized predictor which hampers the biological meaning of the results. Another problem is the prevalence dependency of precision and recall. Further work should be done to identify an optimal strategy. Finally, our method is characterized by the employment of window sizes that are above the normative length of an epitope to optimize performance, which may imply that reported antigenic determinants are not sufficient information for prediction. Notwithstanding, this limits the usefulness of the tool for short sequences or evaluating multiple epitope sites for a given sequence, which enhances accuracy in NetH2pan ${ }^{15}$ or MHCflurry ${ }^{16}$. However, as NAP$\mathrm{CNB}$ is intended to be employed in its complete pipeline form, this a trade-off against providing a single and more robust score to the user.

The variant calling process poses further challenges. Our approach has prioritized a procedure that functions solely on RNA-Seq data with a conservative selection of mutations, particularly missense SNV. This neglects a high percentage of variants that produce neoantigens ${ }^{48}$ and increases the mutational uncertainty by not including genomic data from DNA-Seq ${ }^{21}$. Advances should proceed in this direction, albeit prioritizing an exclusive RNA-Seq utilization to retain the tool's cost-effectiveness, which is essential for our open web service to remain reachable. 
Received: 5 February 2021; Accepted: 27 April 2021

Published online: 24 May 2021

\section{References}

1. Schumacher, T. N. \& Schreiber, R. D. Neoantigens in cancer immunotherapy. Science 348, 69-74. https://doi.org/10.1126/science. aaa4971 (2015).

2. Waldman, A. D., Fritz, J. M. \& Lenardo, M. J. A guide to cancer immunotherapy: From T cell basic science to clinical practice. Nat. Rev. Immunol. https://doi.org/10.1038/s41577-020-0306-5 (2020).

3. Hundal, J. et al. pVAC-Seq: A genome-guided in silico approach to identifying tumor neoantigens. Genome Med. 8, 1-11. https:// doi.org/10.1186/s13073-016-0264-5 (2016).

4. Richters, M. M. et al. Best practices for bioinformatic characterization of neoantigens for clinical utility. Genome Med. 11, 56. https://doi.org/10.1186/s13073-019-0666-2 (2019).

5. Rubinsteyn, A. et al. Computational pipeline for the PGV-001 neoantigen vaccine trial. Front. Immunol. 8, 1-7. https://doi.org/ 10.3389/fimmu.2017.01807 (2018).

6. Kim, S. et al. Neopepsee: Accurate genome-level prediction of neoantigens by harnessing sequence and amino acid immunogenicity information. Ann. Oncol. 29, 1030-1036. https://doi.org/10.1093/annonc/mdy022 (2018) (Epigenetic modifiers as immunomodulatory therapies in solid tumours).

7. Wang, T.-Y., Wang, L., Alam, S. K., Hoeppner, L. H. \& Yang, R. ScanNeo: Identifying indel-derived neoantigens using RNA-Seq data. Bioinformatics 35, 4159-4161 (2019).

8. Wood, M. A. et al. Neoepiscope improves neoepitope prediction with multivariant phasing. Bioinformatics 36, 713-720. https:// doi.org/10.1093/bioinformatics/btz653 (2019).

9. Bjerregaard, A. M., Nielsen, M., Hadrup, S. R., Szallasi, Z. \& Eklund, A. C. MuPeXI: Prediction of neo-epitopes from tumor sequencing data. Cancer Immunol. Immunother 66, 1123-1130. https://doi.org/10.1007/s00262-017-2001-3 (2017).

10. Mösch, A., Raffegerst, S., Weis, M., Schendel, D. J. \& Frishman, D. Machine learning for cancer immunotherapies based on epitope recognition by t cell receptors. Front. Genet 10, 1141. https://doi.org/10.3389/fgene.2019.01141 (2019).

11. Duan, F. et al. Genomic and bioinformatic profiling of mutational neoepitopes reveals new rules to predict anticancer immunogenicity. J. Exp. Med. 211, 2231-2248. https://doi.org/10.1084/jem.20141308 (2014).

12. Bjerregaard, A.-M., Pedersen, T. K., Marquard, A. M. \& Hadrup, S. R. Prediction of neoepitopes from murine sequencing data. Cancer 68, 159-161 (2019).

13. Hasegawa, T. et al. Neoantimon: A multifunctional R package for identification of tumor-specific neoantigens. Bioinformatics 36, 4813-4816. https://doi.org/10.1093/bioinformatics/btaa616 (2020).

14. Lundegaard, C. et al. NetMHC-3.0: Accurate web accessible predictions of human, mouse and monkey MHC class I affinities for peptides of length 8-11. Nucleic Acids Res. 36, W509-W512. https://doi.org/10.1093/nar/gkn202 (2008).

15. DeVette, C. I. et al. NetH2pan: A computational tool to guide MHC peptide prediction on murine tumors. Cancer Immunol. Res. 6, 636-644. https://doi.org/10.1158/2326-6066.cir-17-0298 (2018).

16. O'Donnell, T. J., Rubinsteyn, A. \& Laserson, U. Mhcflurry 2.0: Improved pan-allele prediction of MHC class I-presented peptides by incorporating antigen processing. Cell Syst. 11, 42-48.e7. https://doi.org/10.1016/j.cels.2020.06.010 (2020).

17. Bhattacharya, R. et al. Evaluation of machine learning methods to predict peptide binding to MHC Class I proteins. bioRxiv https:// doi.org/10.1101/154757 (2017).

18. Lipton, Z. C., Berkowitz, J. \& Elkan, C. A critical review of recurrent neural networks for sequence learning. arXiv preprint arXiv: 1506.00019arXiv:1207.0580 (2015).

19. Sønderby, S. K. \& Winther, O. Protein secondary structure prediction with long short term memory networks. arXiv preprint arXiv:1412.7828 (2014).

20. Hsieh, Y.-L., Chang, Y.-C., Chang, N.-W. \& Hsu, W.-L. Identifying protein-protein interactions in biomedical literature using recurrent neural networks with long short-term memory. In Proceedings of the Eighth International Joint Conference on Natural Language Processing (volume 2: short papers), 240-245 (2017).

21. Xu, C. A review of somatic single nucleotide variant calling algorithms for next-generation sequencing data. Comput. Struct. Biotechnol. J. 16, 15-24. https://doi.org/10.1016/j.csbj.2018.01.003 (2018).

22. Overwijk, W. W. \& Restifo, N. P. B16 as a mouse model for human melanoma. Curr. Protoc. Immunol. 39, 20-1 (2000).

23. Taketo, M. et al. Fvb/n: An inbred mouse strain preferable for transgenic analyses. Proc. Natl. Acad. Sci. 88, 2065-2069 (1991).

24. Taneja, P. et al. MMTV mouse models and the diagnostic values of MMTV-like sequences in human breast cancer. Expert. Rev. Mol. Diagn. 9, 423-440 (2009).

25. Andrews, S. FastQC-A quality control tool for high throughput sequence data. http://www.bioinformatics.babraham.ac.uk/proje cts/fastqc/, citeulike-article-id:11583827 (2010)

26. Dobin, A. et al. STAR: Ultrafast universal RNA-seq aligner. Bioinformatics 29, 15-21. https://doi.org/10.1093/bioinformatics/ bts635 (2013).

27. Broad Institute. Picard toolkit. http://broadinstitute.github.io/picard/ (2019).

28. McKenna, A. et al. The Genome Analysis Toolkit: A MapReduce framework for analyzing next-generation DNA sequencing data. Genome Res. 20, 1297-303. https://doi.org/10.1101/gr.107524.110 (2010).

29. Van der Auwera, G. A. et al. From fastQ data to high-confidence variant calls: The genome analysis toolkit best practices pipeline. Curr. Protoc. Bioinform. 43, 1-33. https://doi.org/10.1002/0471250953.bi1110s43 (2013).

30. Poplin, R. et al. Scaling accurate genetic variant discovery to tens of thousands of samples. bioRxiv https://doi.org/10.1101/201178 (2018).

31. Cirulli, E. T. et al. Screening the human exome: A comparison of whole genome and whole transcriptome sequencing. Genome Biol. https://doi.org/10.1186/gb-2010-11-5-r57 (2010).

32. Coudray, A., Battenhouse, A. M., Bucher, P. \& Iyer, V. R. Detection and benchmarking of somatic mutations in cancer genomes using rna-seq data. PeerJ 6, e5362. https://doi.org/10.7717/peerj.5362 (2018).

33. McLaren, W. et al. The ensembl variant effect predictor. Genome Biol. 17, 122. https://doi.org/10.1186/s13059-016-0974-4 (2016).

34. Bateman, A. et al. UniProt: A hub for protein information. Nucleic Acids Res. 43, D204-D212. https://doi.org/10.1093/nar/gku989 (2015).

35. Trapnell, C. et al. Transcript assembly and quantification by RNA-Seq reveals unannotated transcripts and isoform switching during cell differentiation. Nat. Biotechnol. 28, 511-5. https://doi.org/10.1038/nbt.1621 (2010).

36. Vita, R. et al. The immune epitope database (IEDB): 2018 update. Nucleic Acids Res. 47, D339-D343. https://doi.org/10.1093/nar/ gky1006 (2018).

37. Smith, T. \& Waterman, M. Identification of common molecular subsequences. J. Mol. Biol. 147, 195-197. https://doi.org/10.1016/ 0022-2836(81)90087-5 (1981).

38. Henikoff, S. \& Henikoff, J. G. Amino acid substitution matrices from protein blocks. Proc. Natl. Acad. Sci. USA 89, 10915-10919. https://doi.org/10.1073/pnas.89.22.10915 (1992).

39. Chollet, F. et al. Keras. https://keras.io (2015) 
40. Abadi, M. et al. TensorFlow: Large-scale machine learning on heterogeneous systems (2015). Software available from tensorflow. org.

41. Kidera, A., Konishi, Y., Oka, M., Ooi, T. \& Scheraga, H. A. Statistical analysis of the physical properties of the 20 naturally occurring amino acids. J. Protein Chem. 4, 23-55 (1985).

42. Atchley, W. R., Zhao, J., Fernandes, A. D. \& Drüke, T. Solving the protein sequence metric problem. Proc. Natl. Acad. Sci. 102, 6395-6400 (2005).

43. Liu, W., Meng, X., Xu, Q., Flower, D. R. \& Li, T. Quantitative prediction of mouse class I MHC peptide binding affinity using support vector machine regression (SVR) models. BMC Bioinform. 7, 1-13 (2006).

44. Castle, J. C. et al. Exploiting the mutanome for tumor vaccination. Cancer Res. 72, 1081-1091. https://doi.org/10.1158/0008-5472. CAN-11-3722 (2012).

45. Devlin, J., Chang, M., Lee, K. \& Toutanova, K. BERT: Pre-training of deep bidirectional transformers for language understanding. CoRR arXiv:abs/1810.04805 (2018).

46. Jing, L. et al. Gated orthogonal recurrent units: On learning to forget. CoRR arXiv:abs/1706.02761 (2017).

47. Goodfellow, I. J. et al. Generative adversarial networks (2014). arXiv:1406.2661.

48. Vogelstein, B. et al. Cancer genome landscapes. Science 339, 1546-1558 (2013).

\section{Acknowledgements}

This work was funded by the Spanish Ministry of Economy, Industry and Competitiveness (TEC2016-28052-R, RTC2017-6600-1, SAF2017-84091-R, BFERO2020.04), the Spanish Ministry of Science and Innovation (FPU18/03199, PID2019-109820RB-I00), the "la Caixa" Foundation (LCF/BQ/EU19/11710071), FERO foundation and Centro Superior de Investigaciones Científicas (JAEINT18/EX/0636).

\section{Author contributions}

C.W.C. and R.S.G. contributed equally to this work. C.W.C. and R.S.G. designed the neural networks, assembled the genomic workflow, extracted the datasets and developed the web server with J.R.M. A.M.P. and E.V performed the in vitro experiments, which were sequenced and analyzed by R.S.P. and R.A. C.O.S.S. and A.M.B. provided supervision and funding for the project. All authors discussed the results and commented on the manuscript.

\section{Competing interests}

The authors declare no competing interests.

\section{Additional information}

Supplementary Information The online version contains supplementary material available at https://doi.org/ 10.1038/s41598-021-89927-5.

Correspondence and requests for materials should be addressed to A.M.-B.

Reprints and permissions information is available at www.nature.com/reprints.

Publisher's note Springer Nature remains neutral with regard to jurisdictional claims in published maps and institutional affiliations.

(c) (i) Open Access This article is licensed under a Creative Commons Attribution 4.0 International License, which permits use, sharing, adaptation, distribution and reproduction in any medium or format, as long as you give appropriate credit to the original author(s) and the source, provide a link to the Creative Commons licence, and indicate if changes were made. The images or other third party material in this article are included in the article's Creative Commons licence, unless indicated otherwise in a credit line to the material. If material is not included in the article's Creative Commons licence and your intended use is not permitted by statutory regulation or exceeds the permitted use, you will need to obtain permission directly from the copyright holder. To view a copy of this licence, visit http://creativecommons.org/licenses/by/4.0/.

(C) The Author(s) 2021 